\title{
Ressecção Histeroscópica do Endométrio: Resultados e Fatores de Risco para Insucesso
}

\author{
Hysteroscopic Endometrial Resection: Results and Risk Factors for Failures
}

Frederico Faria Rodrigues, Michelle Soares de Moura, Eduardo Camargo Millen,

José Francisco Dória Ramos, Salete Yatabe, Reginaldo Guedes Coelho Lopes, Umberto Gazi Lippi

\section{RESUMO}

Objetivo: estudar o resultado da ablação endometrial histeroscópica, com o uso de ressectoscópio, em pacientes com sangramento uterino anormal de etiologia benigna e refratária ao tratamento clínico, e relatar os fatores associados aos insucessos.

Métodos: foram avaliados retrospectivamente informações sobre 64 pacientes com sangramento uterino anormal de causa benigna, submetidas à intervenção entre abril de 1994 e fevereiro de 2000. A média de idade foi de 42,9 anos e a paridade média, de 2,6 partos. Foi realizada histeroscopia diagnóstica com biópsia endometrial pré-operatoriamente. Duas pacientes receberam previamente gestrinona, seis danazol e 44 análogo do GnRH. No ato cirúrgico realizou-se a cauterização elétrica do fundo uterino e das regiões cornuais. Depois, com o ressectoscópio, foi removido o endométrio até $1 \mathrm{~cm}$ cranialmente ao orificio interno e na profundidade de 2 a $3 \mathrm{~mm}$ na musculatura. A distensão uterina se fazia com glicina a 1,5\% até há dois anos, quando se passou a usar o manitol a 3\%. O seguimento foi de 11,5 meses em média.

Resultados: estabeleceu-se amenorréia em 31,2\% das pacientes e hipomenorréia em 45,3\%. Houve persistência do sangramento anormal em 23,5\%. Houve uma perfuração uterina na cérvico-dilatação (1,5\%). Os resultados foram melhores nas pacientes com mais de 40 anos de idade, nas multiparas e nas que usaram análogos do GnRH. Nos casos de falhas houve freqüência estatisticamente significante $(p=0,04)$ de miomas submucosos de 46,6\% contra $20,6 \%$ no grupo com hipomenorréia e 20\% no grupo com amenorréia. O uso de análogo do GnRH fez decrescer de modo significante $(p=0,03)$ os insucessos. No grupo de pacientes nos quais se considerou haver falha da cirurgia, 46\% tiveram, ao exame anatomopatológico, endométrio secretor; no grupo com hipomenorréia, 10,3\% tiveram este resultado histológico e no grupo com amenorréia, 10\%, o que não apresentou significância estatística $(p=0,12)$. Conclusão: o método mostrou-se útil para tratar o sangramento uterino anormal de etiologia benigna. Recomenda-se o uso de análogos do GnRH previamente à intervenção. Sugere-se que as pacientes mais jovens, com menor paridade e com diagnóstico pré-operatório de mioma submucoso recebam atenção especial, pois entre elas ocorrem falhas com maior freqüência.

PALAVRAS-CHAVE: Sangramento uterino anormal. Histerectomia. Ablação endometrial. Histeroscopia.

\section{Introdução}

O sangramento uterino anormal (SUA), definido como qualquer desvio da normalidade do ciclo menstrual, é problema que afeta uma grande quantidade de mulheres no menacme, e seu tra-

Setor de Endoscopia Ginecológica do Serviço de Ginecologia e Obstetrícia do Hospital do Servidor Público Estadual

"Francisco Morato de Oliveira" - São Paulo - SP

Correspondência:

Umberto Gazi Lippi

Rua Borges Lagoa, 564, cj 32 - Vila Clementino

04038-000 - São Paulo - SP tamento, muitas vezes, um desafio para o ginecologista. Não é recente a tentativa da redução do fluxo menstrual por meio de métodos que destroem o endométrio. Os agentes químicos e fisicos foram abandonados há anos, devido à alta incidência de complicações e falhas terapêuticas. Droegemueller et al. ${ }^{1,2}$ relataram o controle do SUA mediante criocirurgia intra-uterina, mas esta técnica não mostrou bons resultados. Goldrath et al. ${ }^{3}$ descreveram a primeira técnica histeroscópica para a ablação do endométrio, com uso do YAG laser em 22 pacientes portadoras de SUA, cujo resultado foi considerado bom. DeCherney e Polan 4 publica- 
ram os resultados da ablação histeroscópica do endométrio com uso do ressectoscópio em 21 pacientes. Magos et al. ${ }^{5}$ introduziram e sistematizaram esta técnica na Inglaterra, contribuindo para sua ampla divulgação. Atualmente há vários métodos descritos para a ablação endometrial. O mais amplamente utilizado é a ressecção histeroscópica do endométrio, devido à sua segurança, eficácia e menor custo em relação a outros, além de ter um custo até vinte vezes menor que o YAG laser ${ }^{6}$.

Convém ressaltar que até recentemente a histerectomia era o único método eficaz para o controle do SUA refratário ao tratamento clínico, e ainda hoje é muito utilizada para esse fim. Segundo Rutherford ${ }^{7}$, em um estudo realizado na Inglaterra em 1988, 49\% das pacientes submetidas a histerectomia tinham como indicação o SUA de etiologia benigna, sendo que $58 \%$ destas pacientes (ou seja, $25 \%$ do total das histerectomias) não apresentaram qualquer anormalidade no resultado do exame anatomopatológico da peça operatória. Seriam, pois, excelentes candidatas a métodos menos invasivos.

Este estudo tem como objetivo descrever a experiência do Hospital do Servidor Público Estadual "Francisco Morato de Oliveira" - SP (HSPE) com o uso da ressecção histeroscópica do endométrio, no tratamento do SUA de etiologia benigna, nãoresponsivo ao tratamento clínico, colocando em relevo inclusive os insucessos do método.

\section{Pacientes e Métodos}

De abril de 1994 a fevereiro de 2000, 64 pacientes do HSPE-SP foram submetidas a ablação histeroscópica do endométrio, com uso do ressectoscópio. A média de idade das pacientes foi de 42,9 anos (31 a 58). Quanto à paridade, quatro eram nulíparas, e a média de partos foi de 2,6. Todas apresentavam SUA de etiologia benigna, não-responsivo ao tratamento clínico. As pacientes eram informadas previamente da natureza do método, complicações, possibilidade de falha e nãoeficácia do mesmo como contraceptivo, aceitando o procedimento. Foram excluídas pacientes portadoras de alterações neoplásicas malignas ou prémalignas (hiperplasia com atipias) do endométrio, além de pacientes portadoras de cavidade uterina muito aumentada, com histerometria maior do que $12 \mathrm{~cm}$. Realizou-se previamente histeroscopia diagnóstica com biópsia de endométrio em todos os casos. Como medicação pré-operatória foram utilizados gestrinona em duas pacientes, danazol em seis e análogo do GnRH em 44. O tempo de uso do análogo foi, em média, de dois meses. A cirurgia realizou-se uma semana após a segunda dose. Nas demais pacientes (12) não foi utilizada medicação pré-operatória.

Utilizou-se ressectoscópio da marca Stortz ${ }^{\circledR}$ para a remoção do endométrio de toda a cavidade uterina, preservando-se uma região de $1 \mathrm{~cm}$ próxima do orificio interno do canal cervical. Procurouse com isso evitar o colabamento completo da cavidade uterina, o hematometra e a manutenção de um canal para posterior histeroscopia. Inicialmente foi realizada a cauterização do fundo uterino e das regiões cornuais com alça elétrica tipo "roller-ball", com uma potência de 80 a $100 \mathrm{~W}$ obtida a partir de um gerador eletrocirúrgico de alta freqüência. A seguir foi feita a ressecção, sob visualização direta, de todo endométrio até cerca de 2 a $3 \mathrm{~mm}$ de profundidade na musculatura uterina, inicialmente nas paredes posterior, laterais e, por fim, na parede anterior. Depois, com a alça elétrica procedeu-se à hemostasia e à eletrocoagulação de qualquer endométrio remanescente.

O meio de distensão utilizado foi, inicialmente, a glicina a $1,5 \%$, infundida pelo aparelho Hysteromat $^{\circledR}$ de Hamou, com pressão máxima intracavitária de $150 \mathrm{mmHg}$. Desde há dois anos prefere-se o manitol a 3\% como meio de distensão. Nas pacientes com persistência da sintomatologia de sangramento uterino anormal após seis meses da ablação endometrial, foi realizada histerectomia. Utilizou-se o teste monocaudal de Fisher para cálculo de significância estatística.

O tempo de seguimento ambulatorial foi em média de 11,5 meses, variando de dois a 60 . A análise estatística dos dados foi realizada pelo método monocaudal de Fisher.

Resultados

Obteve-se amenorréia em 20 pacientes $(31,2 \%)$, hipomenorréia em $29(45,3 \%)$ e persistência da menorragia em $15(23,5 \%)$. Ou seja, houve sucesso do tratamento em $76,5 \%$ das vezes e falha em $23,5 \%$.

Das pacientes que permaneceram com fluxo inalterado (15), dez foram submetidas posteriormente a histerectomia, duas apresentaram boa resposta ao tratamento clínico pós-operatório, e três não se submeteram a outra forma de tratamento no serviço.

Houve um caso de perfuração uterina durante a cérvico-dilatação $(1,5 \%)$. Esta paciente foi acompanhada com conduta expectante e recebeu alta hospitalar sem outra intercorrência em 48 horas. Uma outra engravidou três meses após a ablação e deu à luz com 38 semanas, por cesárea. Duas apresentaram cefaléia pós-raquianestesia. Não foram observadas complicações adicionais, como hemorragia, infecção ou intoxicação hídrica.

A idade média do grupo em que persistiu o SUA foi de 39,6 anos; naquele no qual a hipomenorréia se estabeleceu, foi de 43,3 e nas pacientes amenorréicas, de 45,9. A paridade média na mes- 
ma ordem de grupos foi de 2,2; 2,9 e 3,4.

Em 46,6\% das pacientes com falha do método havia sido detectada a presença de mioma (submucoso ou intramural) na histeroscopia préoperatória, contra $20,6 \%$ nas que apresentaram hipomenorréia e 20\% no grupo de amenorréia pósoperatória. Estas diferenças mostraram significância estatística pelo teste monocaudal de Fisher $(\mathrm{p}=0,04)$.

Em 46,6\% das pacientes que permaneceram com sangramento uterino inalterado após a cirurgia, foi utilizado o análogo do GnRH no pré-operatório. Nas pacientes que desenvolveram hipomenorréia, 72,4\% utilizaram-no. Nas que tiveram como resultado a amenorréia, 80\%. Estas diferenças mostraram ser estatisticamente significantes pelo teste monocaudal de Fisher $(p=0,03)$.

A presença de endométrio secretor no resultado anatomopatológico do material obtido na cirurgia foi maior no grupo de falha (46\%), quando comparado com o grupo de hipomenorréia $(10,3 \%)$ e amenorréia (10\%), embora a diferença não seja estatisticamente significante $(p=0,12)$. O achado de adenomiose na peça operatória não mostrou diferença com significância estatística entre os grupos $(26,7 \% ; 31 \% ; 30 \%)$.

\section{Discussão}

A ressecção histeroscópica do endométrio está atualmente bem estabelecida como uma alternativa à histerectomia, no tratamento do SUA rebelde ao uso de medicação, conforme ficou demonstrado pelo "Aberdeen Endometrial Ablation Trials Group"8. Após quatro anos da comparação entre histerectomia, ressecção histeroscópica do endométrio (RE) e ablação a laser, esse grupo demonstrou que a histerectomia foi evitada em $76 \%$ das pacientes submetidas ao procedimento.

Os resultados aqui expostos estão próximos a alguns encontrados na literatura. Goldrath et al. ${ }^{3}$ utilizando o YAG laser em 260 pacientes, obtiveram 48\% de amenorréia, 48\% de hipomenorréia, $2 \%$ de fluxo normal e $2 \%$ de falha. Loffer ${ }^{9}$ em 60 pacientes, também utilizando o YAG laser, obteve 20, 49, 20 e 11\%, respectivamente. Donnez ${ }^{10}$ em 50 mulheres, obteve 34, 60, 4 e 2\%. Hamou ${ }^{11}$ em 177 pacientes, com o ressectoscópio, teve 90\% de hipomenorréia e $10 \%$ de falha.

O número reduzido de complicações ${ }^{12}$ é uma das grandes vantagens em relação à histerectomia, além da rápida recuperação e retorno às atividades da paciente e do menor custo, calculado em cerca da metade daquele de uma histerectomia ${ }^{13}$. As complicações mais comuns relatadas na literatura são hemorragia $(0,97$ a 3,5\%), perfuração uterina $(0,64$ a $2,45 \%)$, perfuração uterina com lesão visceral $(0,07$ a 0,08\%), overload, definido como sobrecarga de lí- quido maior que 2 litros (1 a 5\%) e overload com repercussão clínica $(0,47$ a $0,54 \%$ dos casos).

Foram relatados dois casos de morte nos estudos levantados (em uma casuística de 10.896 procedimentos), uma em razão de edema cerebral associado a astrocitoma maligno durante uma ressecção endometrial e outro caso de septicemia três semanas após uma ressecção endometrial. Portanto, a taxa de óbito de 2 a $3 / 10.000^{12}$ para este procedimento é bem inferior à da histerectomia vaginal, de 13 a 26/10.000, e da histerectomia abdominal ${ }^{14}$, de 17 a 50/10000. Complicações a longo prazo (1,25 a 4,58\%) foram: endometrite, pneumonia, histerectomia, laparotomia e embolia pulmonar. A única complicação ligada diretamente ao procedimento observada na casuística aqui apresentada está dentro dos padrões acima expostos.

No que diz respeito às falhas do método, foi uma observação interessante a de que pacientes mais jovens tiveram piores resultados do que aquelas com maior idade. Este achado é convalidado por vários autores ${ }^{15-17}$ sugerindo que este método apresenta seus melhores resultados na perimenopausa. Foi ainda mostrada uma tendência a melhores resultados quanto maior a paridade, embora este fato não seja relatado por outros autores.

Também é importante assinalar a associação entre o diagnóstico de mioma submucoso ou intramural na histeroscopia pré-operatória como um fator associado à falha do método. Esses achados não são contra-indicação formal à prática da ressecção endoscópica, mas é necessário alertar as pacientes quanto às possibilidades de falhas e manter um seguimento pós-operatório rigoroso.

A observação da associação estatisticamente significante do uso de análogos do $\mathrm{GnRH}$ não foi notada por outros autores ${ }^{18,19}$, embora o uso desta medicação seja relacionado a uma redução do tempo operatório, diminuição da absorção de líquido e menor sangramento intra-operatório.

A maior freqüência de endométrio secretor no resultado anatomopatológico das peças operatórias no grupo de falha, em relação aos outros, mostra a importância de se realizar a ressecção do endométrio na primeira fase do ciclo menstrual, evitando a presença de endométrio secretor, espesso, que dificulta o procedimento ${ }^{15-17,20}$. Embora vários autores relacionem a adenomiose à falha da ressecção endoscópica ${ }^{15,17,21}$, na casuística aqui estudada a freqüência de adenomiose não se mostrou diferente entre as pacientes com sucesso cirúrgico ou não. Isto pode ser explicado pela dificuldade do patologista em diagnosticar adenomiose na peça cirúrgica da ablação, na qual se retira apenas uma camada superficial de tecido muscular (1 a $3 \mathrm{~mm}$ ).

Tendo como base o estudo desta casuística inicial pode-se concluir que a ablação endometrial é método de utilidade para o tratamento do SUA refratário ao tratamento clínico. Sugere-se reco- 
mendar o uso de análogos do GnRH previamente à cirurgia, além de cuidados especiais com as pacientes mais jovens, de menor paridade e com diagnóstico histeroscópico pré-operatório de mioma submucoso ou intramural.

\section{SUMMARY}

Purpose: to evaluate the results of 64 hysteroscopic endometrial ablations using a resectoscope in women with abnormal uterine bleeding of benign etiology and nonresponsive to clinical measures and to describe the failures and their associated conditions.

Methods: sixty-four patients with abnormal uterine bleeding were submitted to the intervention between April 1994 and February 2000. The mean age was 42.9 years and the mean parity, 2.6 deliveries. Diagnostic hysteroscopy and endometrial biopsy were performed preoperatively. Before surgery, two women received gestrinone, six danazol and 44 GnRH analogue. During the surgery electric cauterization of the fundus uteri and cornual regions was made. Afterwards, with a resectoscope, the endometrium was removed as far as $1 \mathrm{~cm}$ above the internal ostium, and as deep as 2 to $3 \mathrm{~mm}$ into the myometrium. The uterine distension was obtained with $1.5 \%$ glycine and since two years ago with $3 \%$ manitol. The average follow-up was 11.5 months. After six months of endometrial ablation, the patients with persistence of symptoms were submitted to hysterectomy.

Results: amenorrhea occurred in $31.2 \%$ of the patients and hypomenorrhea in $45.3 \%$. The abnormal bleeding was maintained in $23.5 \%$. There was one uterine perforation during the dilatation of the cervix. Results were better in the higher age and parity ranges and in the women who received GnRH analogues $(p=0.03)$. Where the method was not successful there was a statistically significant frequency of submucous myoma $(p=0.04)$ and a nonsignificant increase of secretory endometrium $(p=0.12)$.

Conclusions: the method is useful for the management of abnormal uterine bleeding of benign etiology. Previous administration of GnRH analogue is recommended. We suggest a special follow-up for the younger, low parous women, and those with submucous myoma, because they are the group with the poorest results.

KEY WORDS: Abnormal uterine bleeding. Hysterectomy. Endometrial ablation.

\section{Referências}

1. Droegemueller W, Greer BE, Davis JR, Makowski EL, Chvapil M, Pollard A. Cryocoagulation of the endometrium at the uterine cornua. Am J Obstet Gynecol 1978; 131:1-9.

2. Droegemueller W, Greer BE, Makowski E. Cryosurgery in patients with dysfunctional uterine bleeding. Obstet Gynecol 1971; 38:256-8.

3. Goldrath MH, Fuller TA, Segal S. Laser photovaporization of endometrium for the treatment of menorrhagia. Am J Obstet Gynecol
$1981 ; 140: 14-9$.

4. DeCherney A, Polan ML. Hysteroscopic management of intrauterine lesions and intractable uterine bleeding. Obstet Gynecol 1983; 61:392-7.

5. Magos A, Baumann R, Turnbull AC. Transcervical resection of the endometrium in women with menorrhagia. BMJ 1989; 298:1209-12.

6. Maher PJ, Hill DJ. Transcervical endometrial resection for abnormal uterine bleeding - report of 100 cases and review of the literature. Aust N Z J Obstet Gynaecol 1990; 30:357-60.

7. Rutherford AJ. Patient selection for hysteroscopic endometrial resection. Br J Obstet Gynaecol 1991; 98:228-30.

8. Aberdeen Endometrial Ablation Trial Group. A randomized trial of endometrial ablation versus hysterectomy for the treatment of dysfunctional uterine bleeding: outcome at 4 years. Br J Obstet Gynaecol 1999; 106:360-6.

9. Loffer FD. Laser ablation of the endometrium. Obstet Gynecol Clin North Am 1988; 15:77-89.

10.Donnez J. Endometrial ablation in dysfunctional bleeding: size of the uterine cavity In: Donnez J, Nisolle M, editors. An Atlas of Laser Operative Laparoscopy and Hysteroscopy. $1^{\text {st }}$ ed. New York: Parthenon; 1994. p 313-22.

11.Hamou JE. Hysteroscopy and microcolposcopy: text and atlas. $1^{\text {st }}$ ed. New York: Appleton \& Lange; 1991.

12.Overton C, Hargreaves J, Maresh M. A national survey of the complications of endometrial destruction for menstrual disorders: the MISTLETOE study. Br J Obstet Gynaecol 1997; 104:1351-9.

13.Sculpher MJ, Bryan S, Dwyer N, Hutton J, Stirrat GM. An economic evaluation of transcervical endometrial resection versus abdominal hysterectomy for the treatment of menorrhagia. Br J Obstet Gynaecol 1993; 100:244-52.

14.Wingo PA, Huezo CM, Rubin GL, Ory HW, Peterson HB. The mortality risk associated with hysterectomy. Am J Obstet Gynecol 1985; 152:803-8.

15. Magos AL, Baumann R, Lockwood GM, Turnbull AC. Experience with the first 250 endometrial resections for menorrhagia. Lancet 1991; 337:1074-8.

16.Baggish MS, Sze EH. Endometrial ablation: a series of 568 patients treated over an 11-year period. Am J Obstet Gynecol 1996; 174:908-13.

17.Garry R, Shelley-Jones D, Mooney P, Phillips G. Six hundred endometrial laser ablations. Obstet Gynecol 1995; 85:24-9.

18.Garry R, Khair A, Mooney P, Stuart M. A comparison of goserelin and danazol as endometrial thinning agents prior to endometrial laser ablation. $\mathrm{Br} \mathrm{J}$ Obstet Gynaecol 1996; 103:339-44.

19.VanDamme JP. One stage endometrial ablation: results in 200 cases. Eur J Obstet Gynecol Reprod Biol 1992; 43:209-14.

20.Deus JM, Coelho Júnior ER, Martins JA, Lopes RGC, Viscomi FA. Ablação histeroscópica do endométrio: análise preliminar de 21 casos. Rev Bras Ginecol Obstet 1994; 16:7-11.

21.Neis KJ, Brandner P. Adenomyosis and endometrial ablation. Gynaecol Endosc 2000; 9:141-5. 